\title{
AN ANALYTICAL APPROACH TO ANALYZING SPACECRAFT FORMATION-FLYING MOTION
}

\author{
Heng Zhang Bin $\mathrm{Xu}$ \\ Institute of Mechanics \\ Chinese Academy of Sciences \\ Beijing 100080, P. R. China
}

\author{
Zhen Min Zhang \\ Institute of Aerospace Technology \\ Harbin University of Technology \\ Harbin150001, P. R. China
}

\begin{abstract}
An analytical approach to analyzing spacecraft formation-flying motion is proposed in this paper. Flight trajectory of spacecraft relative motion related to initial values is analytically described, and several mainly relative motion modes between formation and reference spacecrafts are significantly characterized. On the basis of analytics, furthermore, a new simplified formula is given to be applied to the formation-flying design.
\end{abstract}

\section{Introduction}

In recent years formation-flying design with a group of spacecrafts to execute special missions has become a most important research area ${ }^{1,2,3}$. While all disturbances among spacecrafts are neglected, it can be simplified as the study of multiple two-body dynamics problem. In fact, some research results about two-body motion for spacecraft rendezvous were presented ${ }^{4,5}$. One of them is so called Hill's approach ${ }^{6}$ (or Clohessy-Wiltshire equation) by which the relative motion equation of two spacecrafts is linearized and then the approximated solution is applied to the control design. Recently, Hill's approach has been generalized to formation-flying design and many formation-flying models have been provided ${ }^{7,8,9}$.

The formation-flying problem, however, differs from rendezvous to a great extent. In formation-fly some stable relative geometric continuities should be kept during the whole mission life, but in the latter problem only a short control or operating activity (about $5 \% \sim 10 \%$ of the orbit period) is needed to be considered. Therefore, it is absolutely necessary to more deeply analyze the precise characteristics of relative motion between spacecrafts .

In this paper, the analytical solution of relative motion between the both of formation-flying and reference spacecrafts is directly established by means of geometrically mapping. And several typical behavior modes of relative motion trajectory under different orbit conditions are discussed in some detail. Furthermore, a new simplified formula is derived by parametrical approximation on the basis of this analytical solution.
It can be much conveniently applied to the passive formation-flying design.

\section{Analytical Solution of Spacecraft $\underline{\text { Relative Motion Equation }}$}

In multiple spacecraft formation-flying one key question is needed to definitely describe the relative motion trajectories between every formatting spacecraft $S_{f}$ and the reference spacecraft $S_{r}$ (may be virtual). If all influence forces among spacecrafts are neglected, the formation-flying design could be simplified as the study of the relative motion of multiple two-body, i.e., the relative motion of someone formatting spacecraft $S_{f}$ and reference spacecraft $S_{r}$.

Assume that the reference spacecraft $S_{r}$ runs in a circular orbit around Earth with radius of $R_{0}$, and the formation-fly spacecraft $S_{f}$ runs in an elliptical orbit also around Earth with ellipticity of $e$ and average radius of $R_{f}$, respectively. To Analyze the geometric relationship between $S_{f}$ and $S_{r}$, two coordinate systems, i.e., the Earth-centric coordinate system $O X Y Z$ and the relative coordinate system oxyz, should be set up firstly (see Fig.1).

In $O X Y Z$, let plane $O X Y$ be the same as the orbital plane of $S_{r}$ and axis $O Z$ be the same as the direction of the normal line of reference orbital plane, axis $O X$ is the link direction from Earth-center $O$ to the ascending-cross point of $S_{f}$ arriving at the orbital plane of $S_{r}$, such that $O Y$ can be additionally determined by right-hand rule.

In relative motion coordinate system oxyz , furthermore, the origin $o$ is defined as the mass center of reference spacecraft $S_{r}$, axis $o x$ as the radiusgrowing direction of reference spacecraft orbit, and let axis $o y$ be the same as the flying direction of $S_{r}$, then the third axis $o z$ is also established by right-hand rule.

Now suppose that all disturbed forces are neglected, the relative motion equation between the formation- 
flying spacecraft $S_{f}$ and reference spacecraft $S_{r}$ in relative coordinate system oxyz can be familiarly written as follows:

$$
\left\{\begin{array}{l}
\ddot{x}-2 \omega_{r} \dot{y}-\left(\omega_{r}^{2}-\frac{\mu}{R^{3}}\right)\left(R_{0}+x\right)=0 \\
\ddot{y}+2 \omega_{r} \dot{x}-\left(\omega_{r}^{2}-\frac{\mu}{R^{3}}\right) y=0 \\
\ddot{z}+\frac{\mu}{R^{3}} z=0 \quad, \mu=3.986 \times 10^{5}
\end{array}\right.
$$

Where, $\omega_{f}^{2}=\mu / R_{f}^{3}$ and $\omega_{r}^{2}=\mu / R_{o}^{3}$ are the angular frequency of spacecraft $S_{f}$ and $S_{r}$, respectively, and $R=\sqrt{\left(R_{0}+x\right)^{2}+y^{2}+z^{2}}$ is the flying radius of $S_{f}$ at time $t$.

Let $\Delta_{i}$ refer to the inclination difference of two orbital planes, and the perigee of $S_{f}$ be the placed angle $\Omega_{0}$ rotated along the flying direction from $O X$ axis in the orbital plane of $S_{f}$. In addition, let the initial phase of spacecraft between $S_{f}, S_{r}$ and axis $O X$ be $\theta_{f 0}+\Omega_{0}$ and $\theta_{r 0}$, respectively, then the relative motion trajectory of two spacecrafts can be accurately determined under all of above definitions.

Theorem 1: The analytical solution of relative motion equation (1) is described by the following:

$$
\left\{\begin{aligned}
x= & \frac{r_{\theta}}{2}\left\{\left(1-\cos \Delta_{i}\right) \cos \left(\theta+\omega_{r} t+\Omega_{0}+\theta_{f 0}+\theta_{r 0}\right)+\right. \\
& \left.+\left(1+\cos \Delta_{i}\right) \cos \left(\theta-\omega_{r} t+\Omega_{0}+\theta_{f 0}-\theta_{r 0}\right)\right\}-R_{0} \\
y= & \frac{r_{\theta}}{2}\left\{-\left(1-\cos \Delta_{i}\right) \sin \left(\theta+\omega_{r} t+\Omega_{0}+\theta_{f 0}+\theta_{r 0}\right)+\right. \\
& \left.+\left(1+\cos \Delta_{i}\right) \sin \left(\theta-\omega_{r} t+\Omega_{0}+\theta_{f 0}-\theta_{r 0}\right)\right\} \\
z= & r_{\theta} \sin \Delta_{i} \sin \left(\theta+\Omega_{0}+\theta_{f 0}\right)
\end{aligned}\right.
$$

Where both of $\theta$ and $r_{\theta}$ satisfies

$$
\begin{aligned}
& \int_{\theta_{f 0}}^{\theta+\theta_{f 0}} \frac{\left(1-e^{2}\right)^{3 / 2}}{(1+e \cos \theta)^{2}} d \theta=\omega_{f} t \\
& r_{\theta}=\frac{1-e^{2}}{1+e \cos \left(\theta+\theta_{f 0}\right)} R_{f}
\end{aligned}
$$

Proof: With the beginning, by arranging and calculating (2) and (3), the followings are discovered:

$$
\begin{aligned}
& \sqrt{\left(x+R_{0}\right)^{2}+y^{2}+z^{2}}=r_{\theta} \\
& \dot{\theta}=\frac{\omega_{f}}{\left(1-e^{2}\right)^{3 / 2}}\left[1+e \cos \left(\theta+\theta_{f 0}\right)\right]^{2} \\
& \ddot{\theta}=-\frac{2 e \omega_{f}^{2}}{\left(1-e^{2}\right)^{3}}\left[1+e \cos \left(\theta+\theta_{f 0}\right)\right]^{3} \sin \left(\theta+\theta_{f 0}\right) \\
& \dot{r}_{\theta}=\frac{e \omega_{f} R_{f}}{\sqrt{1-e^{2}}} \sin \left(\theta+\theta_{f 0}\right) \\
& \ddot{r}_{\theta}=\frac{e \omega_{f}^{2} R_{f}}{\left(1-e^{2}\right)^{2}}\left[1+e \cos \left(\theta+\theta_{f 0}\right)\right]^{2} \cos \left(\theta+\theta_{f 0}\right)
\end{aligned}
$$

To consider whether or not $z^{*}=r_{\theta} \cos \theta$ is one special solution of (1), substitute $z^{*}$ and (4a) into (1), then we have:

$$
\begin{array}{r}
\ddot{z}^{*}+\frac{\mu}{r_{\theta}^{3}} z^{*}=\ddot{r}_{\theta} \cos \theta-2 \dot{r}_{\theta} \dot{\theta} \sin \theta-r_{\theta} \ddot{\theta} \sin \theta+ \\
+\frac{\omega_{f}^{2} R_{f}}{\left(1-e^{2}\right)^{2}}\left[1+e \cos \left(\theta+\theta_{f 0}\right)\right]^{2} \cos \theta
\end{array}
$$

In addition, by substituting formula (4b) - (4e) into the above equality, it is much clear that the right side of (5) equals to zero. With the similar procedure to mentioned above, it can be also simply tested and verified that $r_{\theta} \sin \theta \quad, \quad r_{\theta} \cos \left(\theta+\omega_{r} t\right) \quad, \quad r_{\theta} \sin \left(\theta+\omega_{r} t\right) \quad$, $r_{\theta} \cos \left(\theta-\omega_{r} t\right)$ and $r_{\theta} \sin \left(\theta-\omega_{r} t\right)$ are all the other five special solutions of (1). By using all these six special solutions and all initial parameters (such as $R_{0}, R_{f}, e, \Delta_{i}, \Omega_{0}, \theta_{f 0}$ and $\theta_{r 0}$ ), all correlative coefficients in (2) between three axis-variables and six

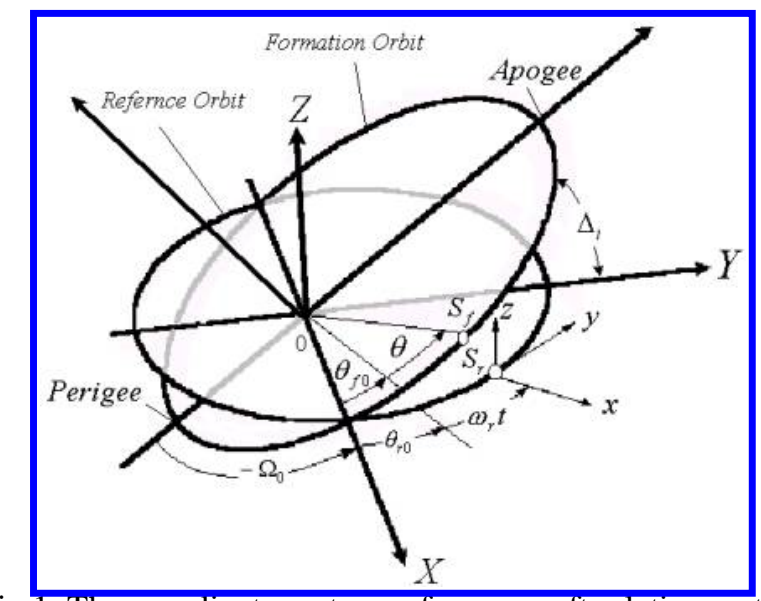

Fig. The coordinate systems of spacecraft relative motion 
special solutions in $o x y z$ can be explicitly obtained . $\Delta$

Clearly, the solution (2) of (1) given by theorem 1 is described by using the relevant orbit and phase parameters of two spacecrafts. If necessary, this solution can be rewritten as the explicit models with six initial values (such as $x_{0}, y_{0}, z_{0} ; \dot{x}_{0}, \dot{y}_{0}, \dot{z}_{0}$ ) in oxyz.

According to $(4 \mathrm{a})$, we have:

$$
\begin{aligned}
& r_{0}=\sqrt{\left(x_{0}+R_{0}\right)^{2}+y_{0}^{2}+z_{0}^{2}} \\
& \dot{r}_{0}=\frac{\dot{x}_{0}\left(x_{0}+R_{0}\right)+\dot{y}_{0} y_{0}+\dot{z}_{0} z_{0}}{\sqrt{\left(x_{0}+R_{0}\right)^{2}+y_{0}^{2}+z_{0}^{2}}}
\end{aligned}
$$

Let $\theta=0$ in (3b) and (4d), and substitute (6a)-(6b) into (3b) and (4d), then we have:

$$
\left\{\begin{array}{l}
e \cos \theta_{f 0}=\left(1-e^{2}\right)\left(\frac{R_{f}}{r_{0}}\right)-1 \\
e \sin \theta_{f 0}=\sqrt{1-e^{2}}\left(\frac{\dot{r}_{0}}{\omega_{f} R_{f}}\right)
\end{array}\right.
$$

For simplicity, define $\lambda_{\theta}=r_{\theta} /\left(\dot{\theta}_{0} r_{0}\right)$. Thus the following can be obtained by $(3 a)-(3 b) 、(4 b)$ and (6a)-(6c):

$$
\begin{aligned}
& e=\sqrt{\left(1-\frac{r_{0}}{R_{f}}\right)^{2}+\left(\frac{r_{0}}{R_{f}}\right)^{2}\left(\frac{\dot{r}_{0}}{\omega_{f} R_{f}}\right)^{2}} \\
& \lambda_{\theta}=\frac{\sqrt{1-e^{2}}\left(\frac{r_{0}}{\omega_{f} R_{f}}\right)}{1+\left[\left(1-e^{2}\right)\left(\frac{R_{f}}{r_{0}}\right)-1\right] \cos \theta-\sqrt{1-e^{2}}\left(\frac{\dot{r}_{0}}{\omega_{f} R_{f}}\right) \sin \theta}
\end{aligned}
$$

$\int_{0}^{\theta} \sqrt{1-e^{2}} \lambda_{\theta}^{2}\left(\frac{\omega_{f} R_{f}}{r_{0}}\right)^{2} d \theta=\omega_{f} t$

Based on the above equality (7a), the relationship of ellipticity and different initial values is shown in Fig.2. Substituting $r_{\theta}=\dot{\theta}_{0} r_{0} \lambda_{\theta}$ into the solution (2), the orbital parameters $\left(\Delta_{i}, \Omega_{0}, \theta_{f 0}, \theta_{r 0}\right)$ corresponding to the relative initial values $\left(x_{0}, y_{0}, z_{0}\right)$ and $\left(\dot{x}_{0}, \dot{y}_{0}, \dot{z}_{0}\right)$ can be solved. Therefore, the following result is derived.

Corollary 1: The explicit solution of (1) with initial parameters $\left(x_{0}, y_{0}, z_{0}, \dot{x}_{0}, \dot{y}_{0}, \dot{z}_{0}\right)$ is described as follows:

$$
\left\{\begin{aligned}
x= & \frac{\lambda_{\theta}}{2}\left\{v_{1} \cos \left(\theta+\omega_{r} t\right)+v_{3} \sin \left(\theta+\omega_{r} t\right)+\right. \\
& \left.+v_{2} \cos \left(\theta-\omega_{r} t\right)-v_{4} \sin \left(\theta-\omega_{r} t\right)\right\}-R_{0} \\
y= & \frac{\lambda_{\theta}}{2}\left\{v_{3} \cos \left(\theta+\omega_{r} t\right)-v_{1} \sin \left(\theta+\omega_{r} t\right)+\right. \\
& \left.+v_{4} \cos \left(\theta-\omega_{r} t\right)+v_{2} \sin \left(\theta-\omega_{r} t\right)\right\} \\
z= & \lambda_{\theta}\left\{v_{5} \cos \theta+v_{6} \sin \theta\right\}
\end{aligned}\right.
$$

where all corresponding coefficient $v_{i}, i=1, \ldots, 6$ is given by the following:

$$
\left\{\begin{array}{l}
v_{1}=-\dot{y}_{0}-\left\{\omega_{r}-\sqrt{1-e^{2}}\left(\frac{R_{f}}{r_{0}}\right)^{2} \omega_{f}\right\}\left(x_{0}+R_{0}\right)+\left(\frac{\dot{r}_{0}}{r_{0}}\right) y_{0} \\
v_{2}=-v_{1}+2 \sqrt{1-e^{2}}\left(\frac{R_{f}}{r_{0}}\right)^{2} \omega_{f}\left(x_{0}+R_{0}\right) \\
v_{3}=\dot{x}_{0}-\left\{\omega_{r}-\sqrt{1-e^{2}}\left(\frac{R_{f}}{r_{0}}\right)^{2} \omega_{f}\right\} y_{0}-\left(\frac{\dot{r}_{0}}{r_{0}}\right)\left(x_{0}+R_{0}\right) \\
v_{4}=-v_{3}+2 \sqrt{1-e^{2}}\left(\frac{R_{f}}{r_{0}}\right)^{2} \omega_{f} y_{0} \\
v_{5}=\sqrt{1-e^{2}}\left(\frac{R_{f}}{r_{0}}\right)^{2} \omega_{f} z_{0} \\
v_{6}=\dot{z}_{0}-\left(\frac{\dot{r}_{0}}{r_{0}}\right) z_{0}
\end{array}\right.
$$

Comparing the two expressions (8) and (2), it is evident that six coefficients in (8b) are not absolutely independent, and they are restricted with inclination difference $\Delta_{i}$ of two orbital planes. Simply, the restrictions can be determined as follows:

$$
\begin{aligned}
&\left(v_{1}^{2}+v_{3}^{2}\right)\left(v_{2}^{2}+v_{4}^{2}\right)=\left(v_{5}^{2}+v_{6}^{2}\right)^{2} \\
&=\left(\sqrt{1-e^{2}}\left(\frac{R_{f}}{r_{0}}\right) \omega_{f} R_{f}\right)^{4} \sin ^{4} \Delta_{i} \\
& \sqrt{v_{1}^{2}+v_{3}^{2}}+\sqrt{v_{2}^{2}+v_{4}^{2}}=2 \sqrt{1-e^{2}}\left(\frac{R_{f}}{r_{0}}\right) \omega_{f} R_{f}
\end{aligned}
$$

\section{Relative Motion Analysis and Simplifications}

As mentioned above, the analytical solution (2) or (8) is an explicit description of (1) in a general way. While the orbit of $S_{f}$ satisfies some special conditions, therefore, this solution may be summed up more

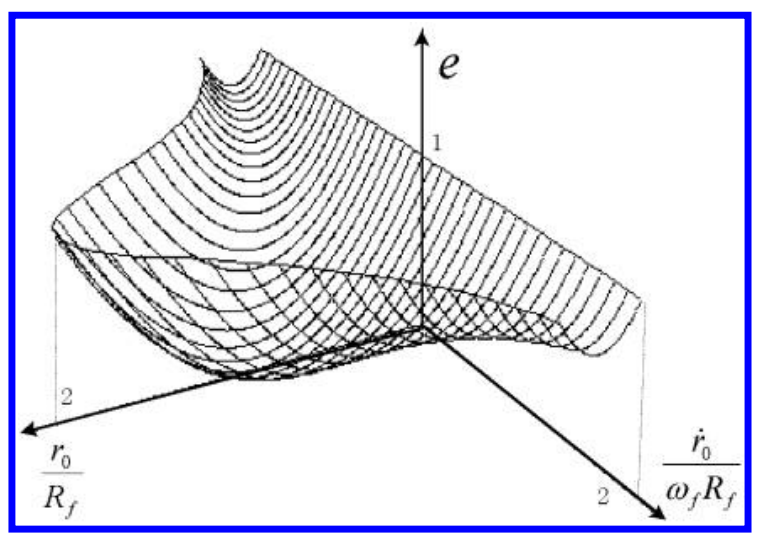

Fig.2 relationship of ellipticity and initial values 
simplified descriptions.

First of all, consider the case of initial values $v_{2}=v_{4}=0$, such that $v_{5}=v_{6}=0$ and $z=0$, such that the following simplification is clearly obtained:

$$
\left\{\begin{array}{l}
x=\frac{r_{\theta}}{r_{0}}\left\{\left(x_{0}+R_{0}\right) \cos \left(\theta+\omega_{r} t\right)+y_{0} \sin \left(\theta+\omega_{r} t\right)\right\}-R_{0} \\
y=\frac{r_{\theta}}{r_{0}}\left\{y_{0} \cos \left(\theta+\omega_{r} t\right)-\left(x_{0}+R_{0}\right) \sin \left(\theta+\omega_{r} t\right)\right\}
\end{array}\right.
$$

In such a way both of $x$ and $y$ is characterized as a group of "high frequency"and big amplitude vibration. From (9) it is also known that this case is corresponded with that $S_{f}$ flies in the direction being opposite of $S_{r}$ (i. e., $\Delta_{i}=180^{\circ}$ ), so that the two satellites may rendezvous most fast with such groups of initial values. It should be pointed out, however, this case must be avoided as possible as we can because this rendezvous under a certain subset of initial conditions may be changed as a "enforced hard touch" differing from the common way expected.

Another extreme case is that $v_{1}$ and $v_{3}$ approximate to zero sufficiently. Thus, the two orbital planes are so closed adequately and two spacecrafts flies in the so almost same direction that a "soft touch on" mode will occur, therefore all responses of $x$ and $y$ are slow enough and the part $\mathrm{z}$ is very closed to zero. In fact, if $S_{f}$ flies in a much low elliptic orbit (i.e. the $2^{\text {nd }}$ order amount of ellipticity $e$ may be neglected), this case will be resulted in under very closed inclination difference. On the basis of this, here a simplified description is represented for formation-flying design.

Theorem 2 ${ }^{10}$ : If $e^{2}$ and $\Delta_{i}^{2}$ are neglected, and $\omega_{f}=\omega_{r}$, then the $1^{\text {st }}$-order approximate solution of (1) is shown as follows

$$
\begin{aligned}
x= & x_{0}+\frac{\dot{y}_{0}}{2 \omega_{r}}+\frac{3}{2}\left(\frac{\dot{r}_{0}}{\omega_{r} R_{0}}\right) y_{0}-\left\{\frac{\dot{y}_{0}}{2 \omega_{r}}+\frac{3}{2}\left(\frac{\dot{r}_{0}}{\omega_{r} R_{0}}\right) y_{0}\right\} \cos \omega_{r} t+ \\
& +\frac{\dot{x}_{0}}{\omega_{r}} \sin \omega_{r} t+o\left(e^{2}\right) \\
y= & y_{0}-\frac{2 \dot{x}_{0}}{\omega_{r}}-3\left(\frac{R_{0}}{r_{0}}-1\right) y_{0}+\left\{\frac{2 \dot{x}_{0}}{\omega_{r}}+3\left(\frac{R_{0}}{r_{0}}-1\right) y_{0}\right\} \cos \omega_{r} t+ \\
& +\frac{\dot{y}_{0}}{\omega_{r}} \sin \omega_{r} t+o\left(e^{2}\right) \\
z= & z_{0} \cos \omega_{r} t+\frac{\dot{z}_{0}}{\omega_{r}} \sin \omega_{r} t+o\left(e^{2}\right)
\end{aligned}
$$

Proof: By neglecting the influences of $e^{2}$ and $\Delta_{i}^{2}$, the followings are obtained from (3a)-(3b):

$$
\begin{aligned}
& \theta-\omega_{r} t=2 e\left\{\sin \left(\omega_{r} t+\theta_{f 0}\right)-\sin \theta_{f 0}\right\}+o\left(e^{2}\right) \\
& \frac{r_{\theta}}{r_{0}}=1+e \cos \theta_{f 0}-e \cos \left(\theta+\theta_{f 0}\right)+o\left(e^{2}\right)
\end{aligned}
$$

such that

$$
\begin{aligned}
x= & \frac{r_{\theta}}{r_{0}}\left\{\left(x_{0}+R_{0}\right) \cos \left(\theta-\omega_{r} t\right)-y_{0} \sin \left(\theta-\omega_{r} t\right)\right\}-R_{0} \\
= & x_{0}+e\left(x_{0}+R_{0}\right) \cos \theta_{f 0}+2 e y_{0} \sin \theta_{f 0}- \\
& -\left\{e\left(x_{0}+R_{0}\right) \cos \theta_{f 0}+2 e y_{0} \sin \theta_{f 0}\right\} \cos \omega_{r} t+ \\
& +\left\{e\left(x_{0}+R_{0}\right) \sin \theta_{f 0}-2 e y_{0} \cos \theta_{f 0}\right\} \sin \omega_{r} t+o\left(e^{2}\right)
\end{aligned}
$$

From (8a), moreover, we have:

$$
\dot{x}_{0}=e \omega_{r}\left(x_{0}+R_{0}\right) \sin \theta_{f 0}-2 e \omega_{r} y_{0} \cos \theta_{f 0}+o\left(e^{2}\right) \quad .
$$

By substituting these intermediate results and (6c) into the above equality, then the validity of (10a) is apparently proved. By the same logic procedure as mentioned above, the proofs of (10b) and (10c) are completed.

As well known, another interesting simplification dealing with relative motion is so called Hill's approach ${ }^{6}$ by which the approximated equation of (1) can be solved as follows under the linearization of $1 / R$ at $1 / R_{0}$ :

$$
\left\{\begin{aligned}
x_{h}(t)= & \left(4 x_{0}+\frac{2 \dot{y}_{0}}{\omega_{r}}\right)-\left(3 x_{0}+\frac{2 \dot{y}_{0}}{\omega_{r}}\right) \cos \omega_{r} t+\frac{\dot{x}_{0}}{\omega_{r}} \sin \omega_{r} t \\
y_{h}(t)= & \left(y_{0}-\frac{2 \dot{x}_{0}}{\omega_{r}}\right)+\frac{2 \dot{x}_{0}}{\omega_{r}} \cos \omega_{r} t+\left(6 x_{0}+\frac{4 \dot{y}_{0}}{\omega_{r}}\right) \sin \omega_{r} t- \\
& -\left(6 \omega_{r} x_{0}+3 \dot{y}_{0}\right) t \\
z_{h}(t)= & z_{0} \cos \omega_{r} t+\frac{\dot{z}_{0}}{\omega_{r}} \sin \omega_{r} t
\end{aligned}\right.
$$

Obviously, one main shortage is that there exists one unconvergent term in the part $y_{h}$ of (11). In order to make Hill's solution (11) usable a strong constraint condition $\dot{y}_{0}+2 \omega_{r} x_{0}=0$ has to be held. Second, due to the linearized approximation, another one condition, i. e. $x^{2}+y^{2}+z^{2}=o\left(e^{2}\right)$ has also to be needed. On the contrary, theorem 2 successfully avoids those being deficient in Hill's approach, because of that (10) is derived by parametrically simplifying the analytical solution of (1) instead of itself of the relative motion equation (1). Therefore, the creditability of theorem 2 after simplification is much better guaranteed than that of (11). In addition, theorem 2 can be applied to longdistance formation flying because of no any additive constraint to initial values needed.

To illustrate how to use theorem 2 to design a formation-flying, here a calculating instance for keeping the track of subsatellite point as a circle is introduced $^{11}$ as follows.

Example 1: Let the radius of the reference spacecraft be th same as $7,500 \mathrm{~km}$. For keeping all subsatellite point (i. e., the track mapping of the formation spacecraft on the plane $y-z$ ) drops on a 
circle, the correspondent initial values should satisfy the following ${ }^{11}$ by (10):

$$
\left\{\begin{array}{l}
\dot{x}_{0}=0.5 \omega_{r} y_{0}\left(1-3\left(\frac{R_{0}}{r_{0}}-1\right)\right) \\
\frac{\dot{z}_{0}}{\omega_{r}}= \pm y_{0} \\
z_{0}=\mp \frac{\dot{y}_{0}}{\omega_{r}} \\
\left(\dot{x}_{0}-\omega_{r} y_{0}\right)^{2}+\left(\dot{y}_{0}+\omega_{r} x_{0}+\omega_{r} R_{0}\right)^{2}+\dot{z}_{0}^{2}=\frac{2 \mu}{r_{0}}-\frac{\mu}{R_{0}}
\end{array}\right.
$$

Let $y_{0}=0$, then we have $\dot{x}_{0}=0, \dot{y}_{0}=\omega_{r} z_{0}, \dot{z}_{0}=0$, and $x_{0}$ is determined by using the fourth one of the above equality. Thus three cases under different track radius (equals to $10 \mathrm{~km}, 150 \mathrm{~km}$ and $1000 \mathrm{~km}$, respectively) of subsatellite point are calculated and shown in Fig. 3 (see all real-lines). To compare the difference between the mentioned calculation and Hill's approach, we can use one proposed formula ${ }^{12}$ derived from Hill's solution (11) to get another three groups

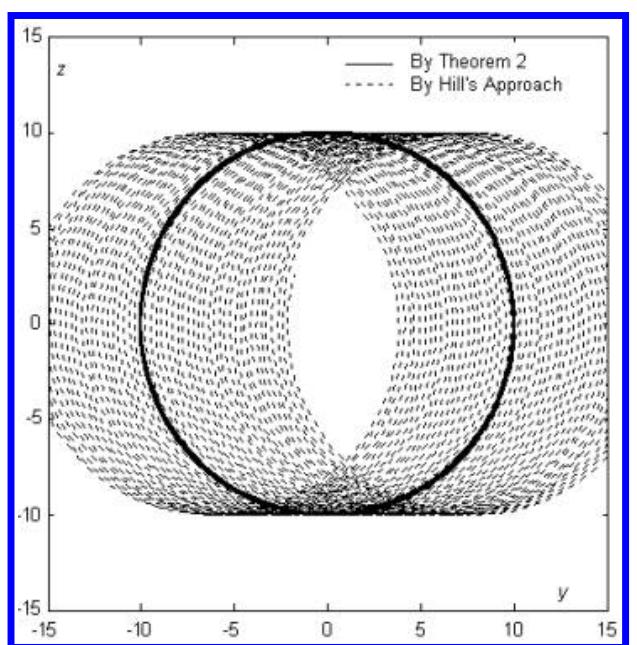

(a) The track radius of subsatellite point $=10 \mathrm{~km}$ of initial values, and all tracks of subsatellite point are drawn in Fig 3 with dush-lines.

Clearly, all tracks of subsatellite point calculated by means of theorem 2 are significantly closed to what designed no matter how the track radius changed. Unfortunately, the designs by Hill's approach indeed result in unconvergent effects, because the mapping tracks can not be periodically repeated.

\section{$\underline{4 . \text { Conclusions }}$}

In this paper the relative motion between formation spacecraft and reference spacecraft orbit has been analytically described in detail. Several response modes of spacecraft relative motion on this basis have been discussed. Furthermore, a new simplified formula better than Hill's approach has been provided under low ellipticity of formation-orbitting and it can be more effectively applied to the spacecraft formation-flying design.
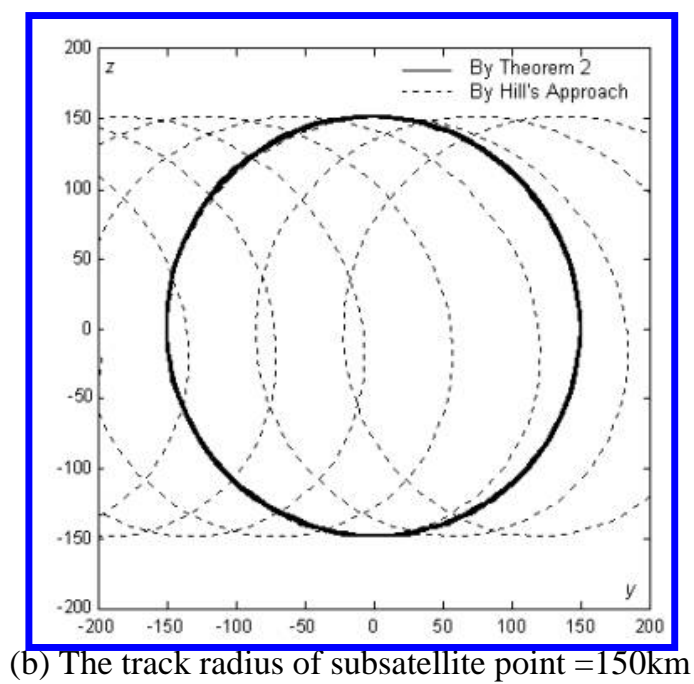

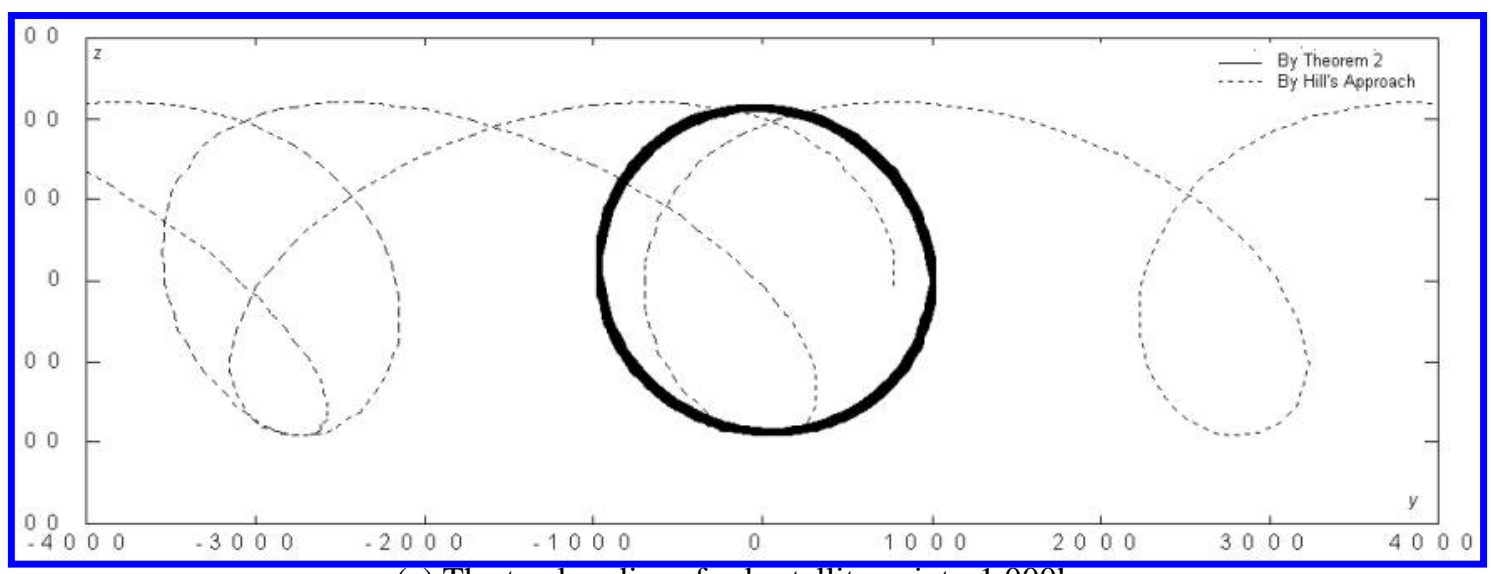

(c) The track radius of subsatellite point $=1,000 \mathrm{~km}$

Fig. 3 The calculated tracks of subsatellite point under three designated cases 


\section{References}

[1] Schaub, H., S. R. Vadali, J. L. Junkins and K. T. Alfriend, Spacecraft Formation Flying Control Using Mean Orbit Elements, The Journal of Astronautical Sciences, Vol. 48, No.1, 2000, pp.69-87.

[2] Milam, M. B., N. Petit and R. M Murray, Constrained Trajectory Generation for MicroSatellite Formation Flying, AIAA 2001-4030, 2001.

[3] Kitts, C., et al, An Experimental Mission in Robust Distributed Space Systems, The AIAA/Utah State University Conference on Small Satellites, 1999.

-[4] Clohessy, W. H and R. S. Wiltshire, Terminal Guidance System for Satellite Rendezvous, Journal of Aerospace Sciences, Vol. 27, No. 9, 1960, pp.653-658.

[5] Carte, T. E, State Transition Matrices for Terminal Reabderzvous Studies: Brief Survey and New Exapmple, Journal of Guidance, Control and Dynamics, Vol. 21, No.1, 1998, pp.148-155.

-[6] Hill, G. W, Researches in the Lunar Theory, American Journal of Mathematics, Vol. 1, No.1, 1878, pp.5-26.

[7] Koon, W. S., J. E. Marsden and R. M. Murray, J2 Dynamics and Formation Flight, AIAA-4090, 2001.

[8] Danielson, D. A., et al, Semianalytic Satellite Theory, Technical Report NPS-MA-95002, Naval Postgraduate School, 1994.

[9] Sabol, C., S. Carter and M. Bir, Analysis of preselected orbit generator options for the Draper Semianalytic Satellite Theory, AIAA 2000-4231, 2000.

[10] Zhang, Z. M., B. Xu and H. Zhang, et al, Analysis of Spacecraft Relative Motion and Applicability of Hill's Solution, Chinese Space Science and Technology, No. 2, 2002, pp. 1-8.

[11] Sun, L., B. Xu and H. Zhang, The Concept and Design Method of Long-distance FormationFlying, Proceedings of the $10^{\text {th }}$ National Annual on Control Technology of Motion body in Space, Zhangjiajie, Hubei, P. R. China, 10-14 Aug, 2002.

[12] Sabol, C., R. Burn and C. McLaughlin, Formation Flying Design and Evolution, $\underline{A A S}$ 99-126, 1999. 